Conference Proceedings Paper

\title{
A Generalized Control Model and Its Digital Algorithm for Aerospace Electrohydraulic Actuators
}

\author{
Zhao Shoujun*, Chen Keqin, Zhang Xiaosha, Zhao Yingxin, Jing Guanghui, Yin Chuanwei, \\ Xiao Xue
}

Beijing Institute of Precision Mechatronics and Controls

No.1 South DaHongMen Road, Fengtai District, Beijing, China, 100076

* Correspondence: shoujunzhao@vip.sina.com; Tel.: +86-10-8852-3021

\begin{abstract}
It is difficult to describe precisely and thus control satisfactorily the dynamics of an electrohydraulic actuator to drive a high thrust liquid launcher engine, whose structural resonant frequency is usually low due to its heavy inertia and its complicated mass distribution. A generalized model is therefore put forward for maximum simplification and sufficient approximation, where a second-order transfer function is used to model the heavy mass-spring nature of the large engine body outside of the rod position loop, another second-order transfer function with two zeros and two poles representing the hydro-mechanical composite resonance effect in the closed rod position loop. A combined control strategy is applied to meet the stringent specification of static and dynamic performances, including a notch filter, a piecewise or nonlinear PID and a feed-forward compensation. The control algorithm is implemented in digital signal processors with a same software structure but different parameters for different aerospace actuators. Compared to other approaches, it is easier this w ay to grasp the system resonance nature, and most importantly, the traditional dynamic pressure feedback is replaced with the convenient digital algorithm, bringing prominent benefits of simplified design, reduced hardware cost and inherent higher reliability. The approach has been validated by simulation, experiments and successful flights.
\end{abstract}

Keywords: control model; control algorithm; electrohydraulic actuators

\section{Introduction}

The electro-hydraulic servo actuation is a well-developed technology. Nonetheless, most of its physical understanding and mathematical modelling have been referred to the Hydraulic Control Systems by H.E. Merritt as early as in 1967 [1]. In most cases, it was assumed that the driven load had sufficiently high structural resonances and $\mathrm{w}$ as modelled as a lumped mass directly attached to the piston rod or motor shaft, with the hydraulic natural frequency dominating the control loop. However, in aerospace applications, such as rudders, fins and engines, due to weight and space limitations, the structural stiffness is usually low and the model has to be modified. It was also discussed in the classic book, where, however, only the dynamics at the motor shaft point was elaborated, with the dynamics at the load end left open for more work [1] (pp.157-162).

An appropriate model for realistic aerospace applications w as presented by J.W. Edward for an aircraft rudder control servo system [2]. A spring $w$ as inserted as the structural compliance into the interface between the piston rod and the rudder. The structural natural frequency, the hydraulic natural frequency, and the derived composite hydro-mechanical natural frequency, were clearly depicted and incorporated into the model, based on which the rudder surface dynamics was controlled by the Dynamic Pressure Feedback (DPF) method and matched well with the test results. The concise approach has been accepted so far [Karl Eric Rydberg] (pp.35-37), how ever, not widely enough, as seen by the author. In most current publications on electro-hydraulics, only the hydraulic resonance is included [4][5]. As well, in most papers studying various emerging electro-hydrostatic 
actuators, where there are also hydraulic cylinders, there is only the hydraulic natural frequency considered [6][7]. This approach might work well somewhere but not in highly dynamic aerospace actuators. Moreover, since the digital control has been the first choice now adays, a better model is greatly helpful to understand the underlying physics, to fabricate the control algorithm and to accelerate the development process.

The author team has been working on electro-hydraulic actuators to gimbal Chinese non-toxicnon-pollution launcher engines [8][9][10]. In practice, the easy approach by J.W. Edwards has been used and developed with a new application perspective and more details, w orthy to be elaborated here. For a variety of actuation systems, a normalized model was developed, in the easy form of control block diagram w ritten in transfer functions. A one-mass-one-spring model of the structural resonance, including the natural frequency and the damping ratio, as well as its measurement, was described. Inside the piston position loop, there w as another second-order transfer function $w$ ith two zeros and two poles representing the hydro-mechanical composite resonance effect. It was pointed out that the poorly damped and low frequency structural load resonance is the root cause of the poor system stability, rather than the hydraulic resonance as mostly blamed. A combined control strategy of notch filter, nonlinear PID and feed-forward compensation was given to meet the stringent requirement of static and dynamic performances. Another two-mass-two-spring model was introduced to deal with the thrust vector control of a heavy launcher engine with a more complex mass and stiffness distribution.

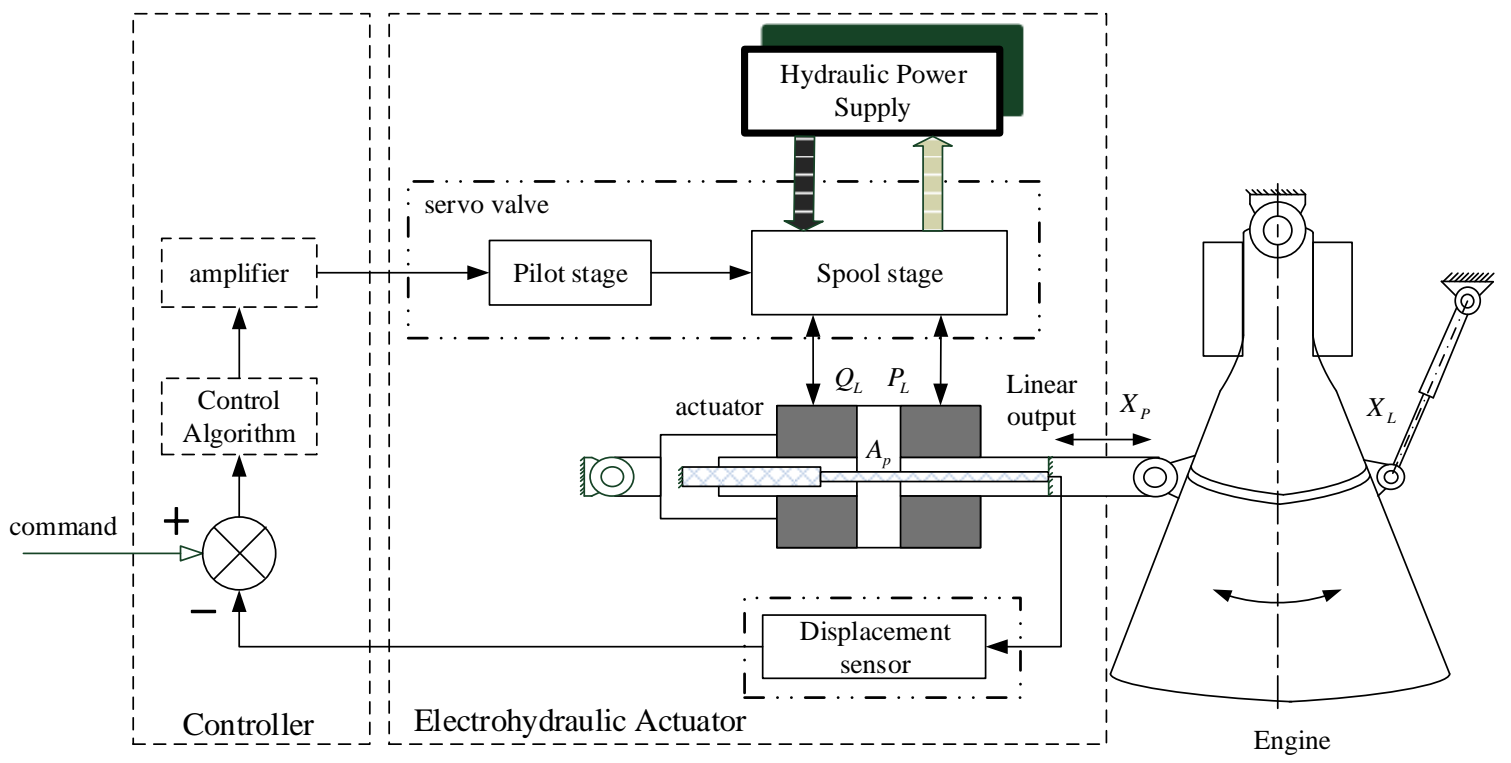

Figure 1. The simplified schematics for an aerospace electrohydraulic actuation system

\section{The System}

A simplified schematics of an aerospace electrohydraulic actuation system to drive a launcher engine is described as in Fig.1. The control loop includes a digital controller, a servo-valve, a double acting piston actuator and a linear displacement sensor imbedded inside the rod. The controller closes the negative feedback loop and performs digital algorithms for static and dynamic compensations. The expected output is the engine's gimbaling angle. It is a classic aerospace design that the feedback signal is picked up via the sensor inside the piston rod rather than via the angular output sensor.

\section{The System Model}

The physical model to connect the actuator and the engine is critical. A one-mass-one-spring model is precise enough to depict most engine dynamics as in Fig.2, since the gimbaling inertia is the dominating load. 


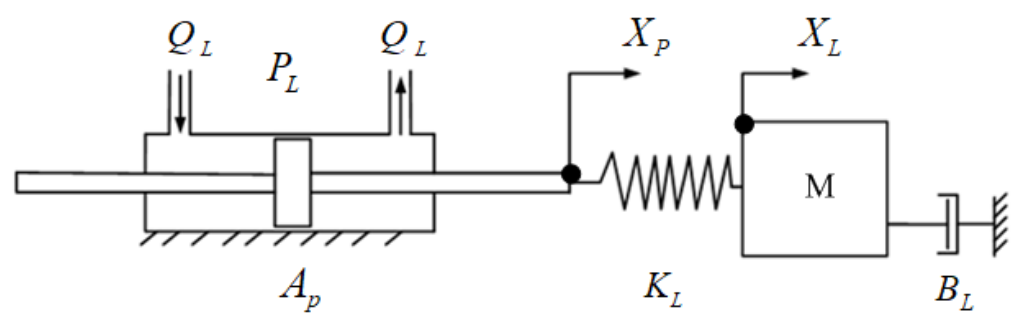

Figure 2. The physical model of a hydraulic actuator to drive an engine load

Though its simplicity is a surprise, it is notew orthy that, in reality, there is neither a real connection spring between the rod and the engine nor a support spring at the actuator body end, despite that a real spring exists in most load simulators. It $w$ as found to precisely depict the internal mass distribution inside the engine body and hence its resonance dynamics. In practice, the system is designed such that other structures, like the piston rod, the actuator body and its fixed supporting structure, are so strong that only the stiffness inside the load needs to be modelled.

Once the physics is clear, the mathematical equations are presented as from Eq. (1) to (4). The symbols are listed in Appendix A.

$$
\begin{gathered}
Q_{L}=A_{p} \cdot S \cdot X_{p}+\frac{v_{t}}{4 \beta} \cdot S \cdot P_{L}+K_{c} \cdot P_{L} \\
A_{p} \cdot P_{L}=K_{L} \cdot\left(X_{p}-X_{L}\right) \\
K_{L} \cdot\left(X_{p}-X_{L}\right)=M \cdot S^{2} X_{L}+B_{L} \cdot S \cdot X_{L} \\
M=\frac{J}{R^{2}}
\end{gathered}
$$

To derive a normalized model, the first step is that the load dynamics in Eq. (3) has to be changed into a second-order transfer function as Eq. (5).

$$
\begin{gathered}
G_{L}=\frac{X_{L}}{X_{p}}=\frac{1}{\frac{1}{\omega_{L}^{2}} S^{2}+\frac{2 \xi_{L}}{\omega_{L}} S+1} \\
\omega_{L}=\sqrt{\frac{K_{L}}{M}} \\
\xi_{L}=\frac{B_{L}}{2 K_{L}} \sqrt{\frac{K_{L}}{M}}
\end{gathered}
$$

To note, the engine's structural resonant frequency $\omega L$ and the damping ratio $\xi_{L}$ are independent of any other electro-hydraulic actuator design except the installation geometry on which the rotation radius $R$ depends. In ground testing, there are 2 measure points, with one at the piston rod as $X_{p}$ and the other at the engine gimbaling angular output as $X_{L}$. Usually the engine gimbaling angle is measured in the form of linear displacement and converted to the angular value.

The next step is to represent the equations (1)(2)(5) into a control block diagram as Fig.3. 


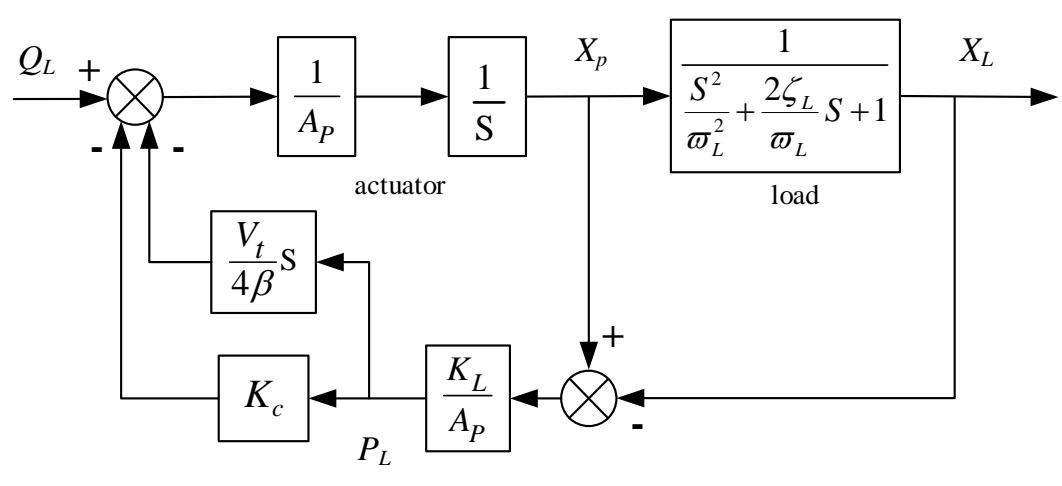

Figure 3. The block diagram of a hydraulic actua tor to drive an engine load

So far, the model derivation in Fig.3 has been a routine and usually stops here. To get a more concise form, it needs to eliminate the minor loops. Though a little tricky and laborious, it is worth seeing the Fig.4 after successful block reductions. With the servo-valve and the controller added as well as a lumped open loop gain included, the final normalized system model is given as Fig.5.

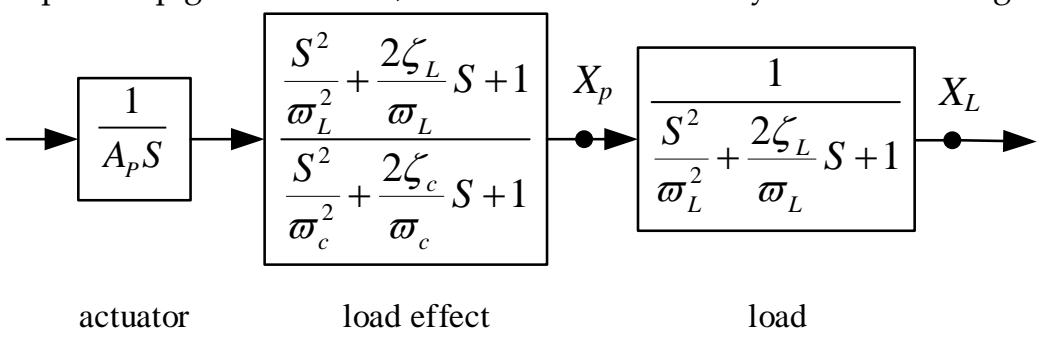

Figure 4. A normalized block diagram of a hydraulic actuator to drive an engine load

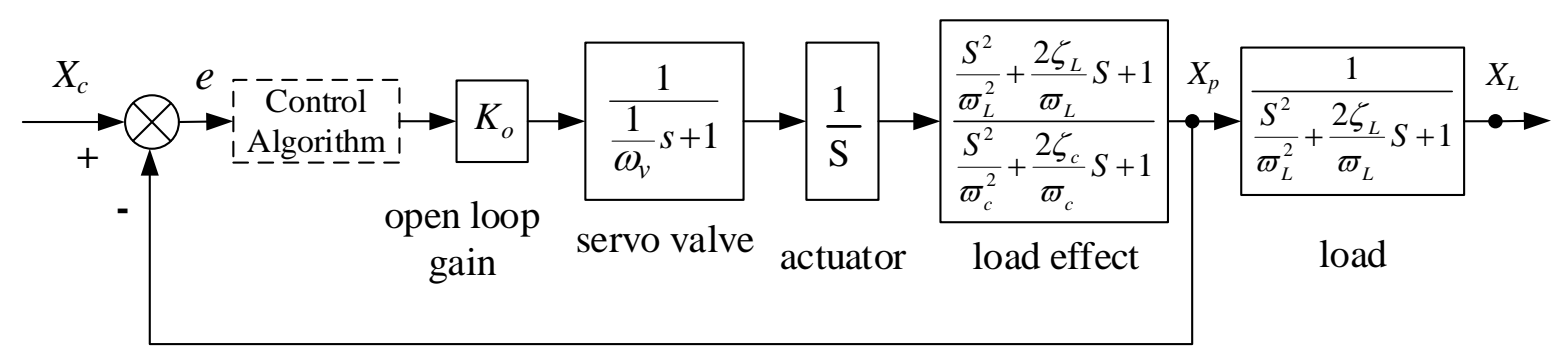

Figure 5. A normalized block diagram of an a erospace electrohydraulic actuation system

The servo-valveis modelled as a first order transfer function in that its bandwidth is chosen as much higher than the structural resonance. The other underlying equations are given as from Eq. (8) to Eq. (12).

$$
\begin{gathered}
\omega_{h}=\sqrt{\frac{4 A_{p}^{2} \beta}{M V_{t}}} \\
\xi_{h}=\frac{K_{c}}{A_{p}} \sqrt{\frac{\beta M}{V_{t}}} \\
\frac{1}{\omega_{c}^{2}}=\frac{1}{\omega_{L}^{2}}+\frac{1}{\omega_{h}^{2}}
\end{gathered}
$$




$$
\begin{gathered}
\xi_{c}=\left(\frac{\xi_{L}}{\omega_{L}}+\frac{\xi_{L} \cdot K_{L}}{\omega_{L} \cdot A_{p}^{2}}+\frac{\xi_{h}}{\omega_{h}}\right) \cdot \omega_{c} \\
K_{o}=K_{v i} \cdot K_{q i} \cdot K_{p d} \cdot \frac{1}{A_{p}}
\end{gathered}
$$

As can be seen, as for the expected output $X_{L}$, it is only a half closed loop. Inside the piston position $X_{p}$ loop, a second-order transfer function with two zeros and two poles dominates, both poorly damped, representing the hydro-mechanical composite resonance effect arising from coupling between the load structural resonance and the hydraulic resonance, simply called "load effect".

Since the structure natural frequency $\omega_{L}$ is low, the acting piston area $A_{p}$ has to be large so that the hydraulic natural frequency $\omega_{h}$ is much larger, often at least triple as big $\omega$. The resulted composite frequency $\omega_{c}$ in the denominator is a little smaller than the load natural frequency $\omega L$.

Inside the loop, the second-order numerator is helpful to cancel out the hydro-mechanical resonance effect of the second-order denominator. However, there is still another second-order denominator outside the loop, representing the load structural resonance. Therefore, both inside and outside theloop, there are two second-order denominators but only one second-order numerator. As a result, at least another second-order numerator is needed for the system stability. Without any compensation, a higher open loop gain would definitely lead to serious vibrations. In the realistic world, there should be disastrous resonance to destroy structures.

In a word, the poorly damped and low frequency structuralload resonance is the root cause of the poor system stability, rather than the hydraulic resonance as mostly blamed.

\section{The Control Algorithm}

A combined control strategy was developed as Fig.6.

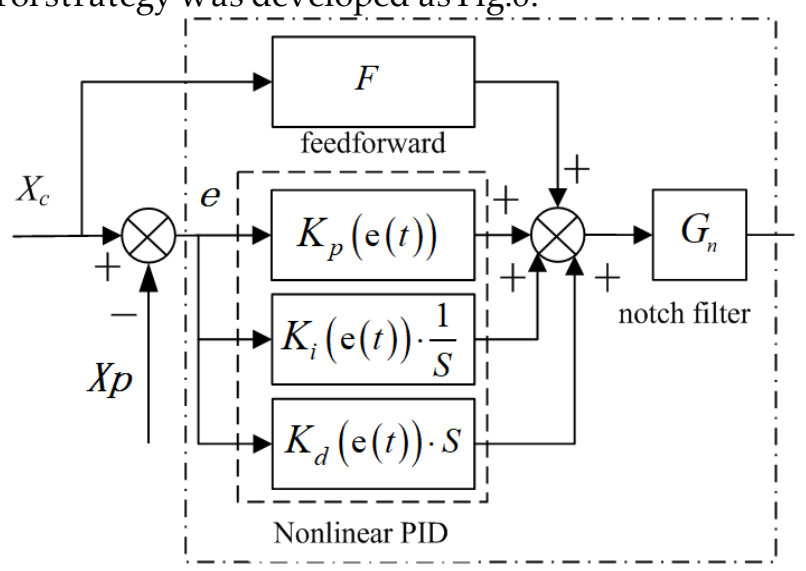

Figure 6. The combined control strategy

The control algorithm comprises three parts: a notch filter, a nonlinear PID, and a feedforward compensation.

The notch filter function $G_{n}$ is used to effectively suppress the system resonance, shown as Eq. (13).

$$
G_{n}=\frac{\frac{1}{\omega_{n 1}^{2}} S^{2}+\frac{2 \xi_{n 1}}{\omega_{n 1}} S+1}{\frac{1}{\omega_{n 2}^{2}} S^{2}+\frac{2 \xi_{n 2}}{\omega_{n 2}} S+1}
$$

The rationale is that the poorly damped poles $\left(\omega_{c}, \xi_{c}\right)$ are cancelled out by a pair of nearly poorly damped zeros $\left(\omega_{n 1}, \xi_{n 1}\right)$ in the numerator and replaced with a pair of better damped poles $\left(\omega_{n 2}, \xi_{n 2}\right)$. It can be as simple as $\omega_{n 1}=\omega_{n 2}=\omega_{c}, \xi_{n 1}=\xi_{c}, \xi_{n 2}=0.2 \sim 0.4$, and usually $\xi_{r} \approx 0.03 \sim 0.1, \xi_{r} \approx \xi_{c}+0.01$. In practice, the parameters can be optimized to change the width and depth of the notch window. 
The nonlinear PID is used to improve the tracking accuracy in the low frequency band, i.e., around $1 \sim 5 \mathrm{rad} / \mathrm{s}$, with a piecewise proportional gain presented as Eq. (14).

$$
K_{P}(e(t))=\left\{\begin{array}{lll}
f_{K} \cdot K_{P} & \left(f_{K}>1\right) & |e(t)| \leq e_{n} \\
K_{P} & |e(t)|>e_{n}
\end{array}\right.
$$

A higher gain near the zero is used to deal with the nonlinearities at the servo-valve spool center. The differential factor is usually small and helps to overcome the stiction forces in the system. As to the integral factor, it helps to improve the positioning precision and needs to be set as active only when the hydraulic power is on so that an integration saturation and thus a jittering at the startup can be avoided.

A reduced-order feedforw ard compensation is given as Eq.15.

$$
F=K_{f} \cdot S \cdot X_{c}
$$

In the intermediate frequency band, some distance from both the high frequency structural resonance region and the low frequency region, a higher open loop gain $K_{0}$ is needed for high precision velocity tracking. How ever, even with a notch filter, there is a limit to increase the magnitude of $K_{o}$, e.g., one third of the structural natural frequency $\omega_{c}$, so that a reduced-order feedforw ard compensation is of a great help to reduce the phage lag in this frequency region. The underlying principle is that, with higher and low er frequency components roughly neglected, there is only one integral transfer function left in the closed loop, which can be cancelled out by a differential counterpart represented as the feedforw ard compensation.

As described above, with a combined control strategy, the system vibration due to structural resonance should be well suppressed while a satisfactory frequency response in the whole frequency region from low to high could be obtained.

\section{Simulations an d Experiments}

An electro-hydraulic servo actuator to drive a high thrust liquid-hydrogen-liquid-oxygen launcher engine is illustrated here, with main parameters show $n$ in Tab. 1.

TABLE 1. The main parameters of the acutation system

\begin{tabular}{cccc}
\hline Parameter & Symbol & Value & Unit \\
\hline Engine rotational inertia & $\mathrm{J}$ & 1000 & $\mathrm{~kg} \cdot \mathrm{m} 2$ \\
Engine rotational arm & $\mathrm{R}$ & 867 & $\mathrm{~mm}$ \\
Equivalent linear mass & $\mathrm{M}$ & 1330 & $\mathrm{Kg}$ \\
Engine natural frequency & $\omega_{L}$ & 96 & $\mathrm{rad} / \mathrm{s}$ \\
Engine natural damping ratio & $\xi_{L}$ & $0.03 \sim 0.1$ & $\mathrm{dimensionless}$ \\
Actuator acting piston area & $\mathrm{A}$ & 4082 & $\mathrm{~mm} 2$ \\
System pressure & $\mathrm{Ps}$ & 21 & $\mathrm{MPa}$ \\
Servovalvebandwidth $\left(-45^{\circ}\right)$ & $\omega_{v}$ & $\geq 180$ & $\mathrm{rad} / \mathrm{s}$ \\
Open loop gain & $K o$ & $10 \sim 30$ & $\mathrm{rad} / \mathrm{s}$ \\
Maximum angular output & - & 4 & $\mathrm{degree}$ \\
Digital Control Cycle & - & 0.001 & second \\
\hline
\end{tabular}

With only an open loop gain of $15 \mathrm{rad} / \mathrm{s}$ and without any other compensation, given a series of sinusoidal commands, the system was tested to study the load resonance effect, with bode plots of $X_{L}$ and $X_{p}$ shown in Fig.7. 


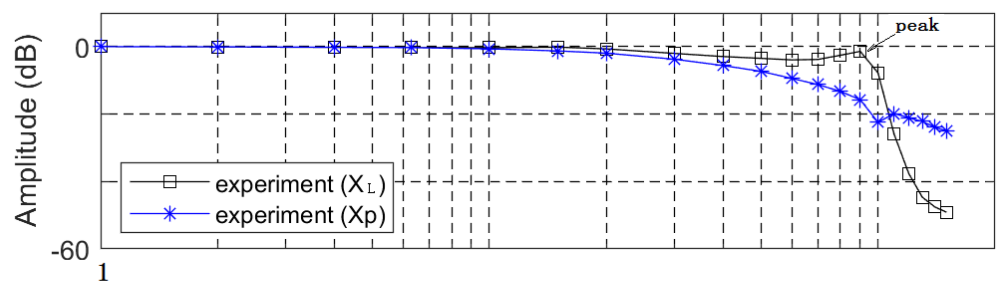

Frequency (rad/sec)

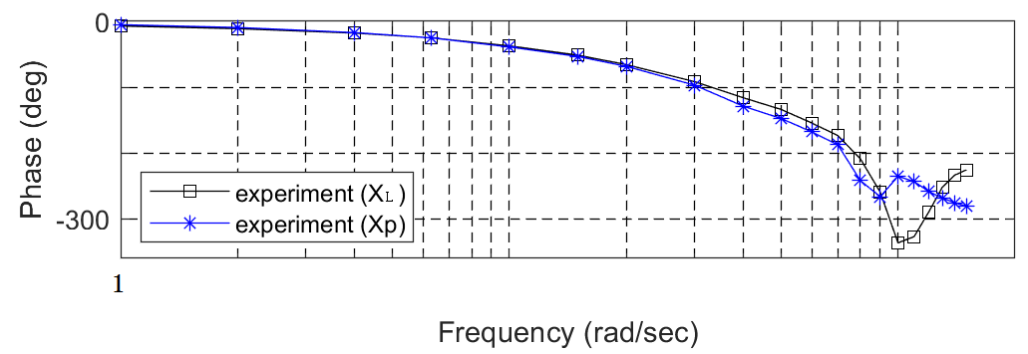

Figure 7. The dynamics of an uncompensated electro-hydraulic actuator and its engine load

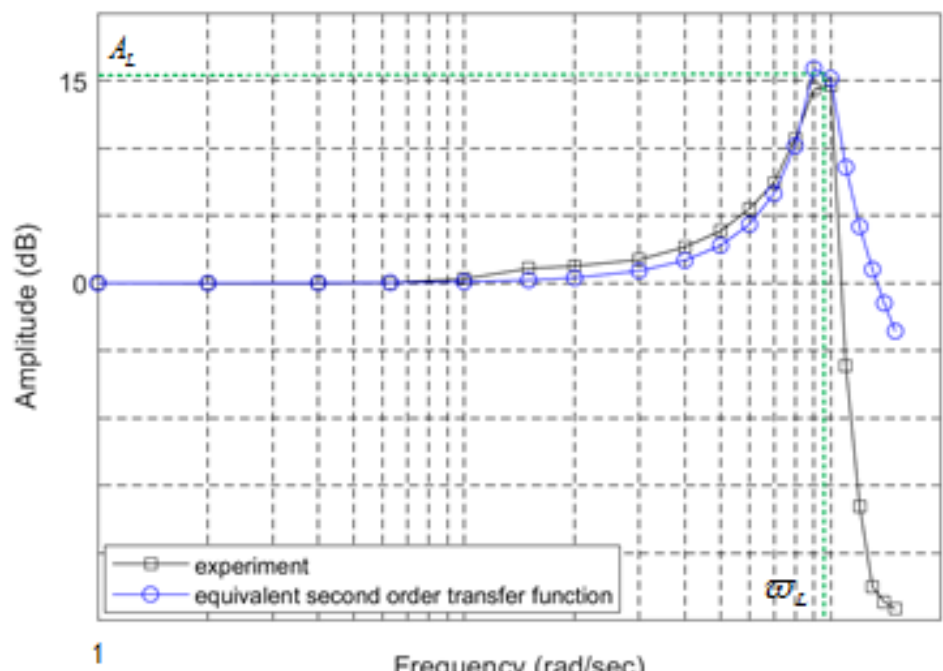

Figure 8. The structuraldyna mics of a liquid-hydrogen-liquid-oxygen launcher engine

It is to note that, without compensation, with a small open loop gain, the system has a tendency to vibrate, as shown by the amplitude peak of the load response $X_{L}$. It is clear that the system has to be compensated for a bigger gain and better dynamics.

The engine's structural resonance dynamics can be obtained by directly subtracting the response of $X_{p}$ at the piston point from that of $X_{L}$ at the load output in Fig.7, resulting a curve shown in Fig. 8, together with a simulation by a standard second-order transfer function.

As shown, the load resonance can be modelled as a second-order transfer function with a sufficient precision. The structural natural frequency $\omega\llcorner$ can be easily identified while the natural damping ratio, $\xi_{L}$ can be computed by Eq.(16).

$$
\xi_{L}=\frac{1}{2} 10^{-\frac{A_{L}}{20}}
$$

As the peak amplitude show $\mathrm{n}$ is $15 \mathrm{~dB}$, accordingly, the damping ratio $\xi_{L}$ is 0.089 .

To observe the hydro-mechanical resonance effect, we calculated the open loop response $X_{p o}$ by breaking the closed loop response $X_{p}$, with the method shown as Eq. (17) and (18), where the response $\mathrm{w}$ as represented in a complex form. The calculated bode plot is given in Fig.9. 


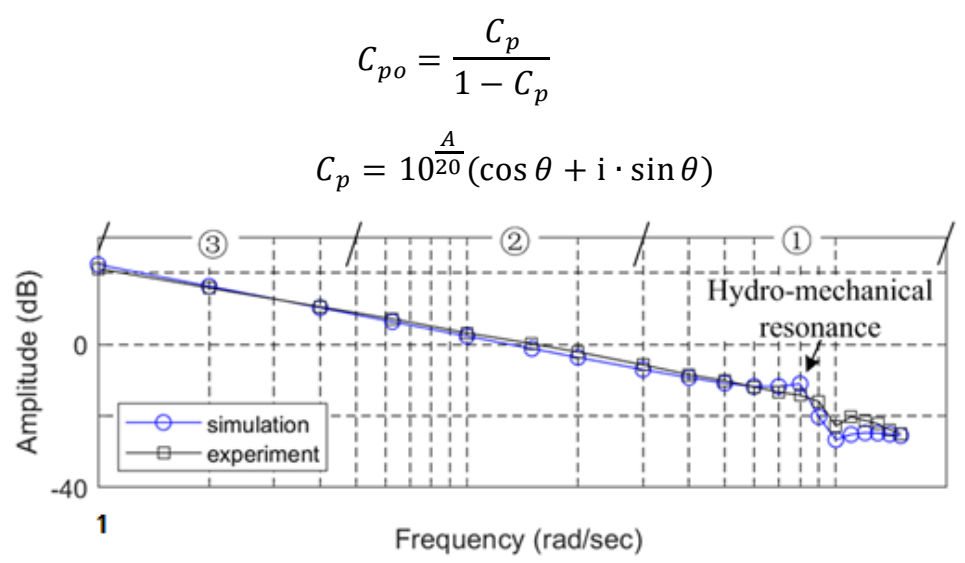

Figure 9. The open loop dynamics of the piston position loop

It can be seen clearly that, the hydro-mechanical resonance peak in the amplitude curve is the bottle neck to increase the stability margin. While the DPF was traditionally used to suppress the resonance, with the digital control at hand, a notch filter is the most straightforw ard and effective approach.

Also as in Fig.9, there are three regions marked to illustrate the combined control strategy. While the notch filter deals with the resonance in the high frequency region (1), a higher gain and the feedforward compensation improve the velocity tracking precision in the intermediate frequency region (2), and the nonlinear PID upgrades the response in the low frequency region (3).
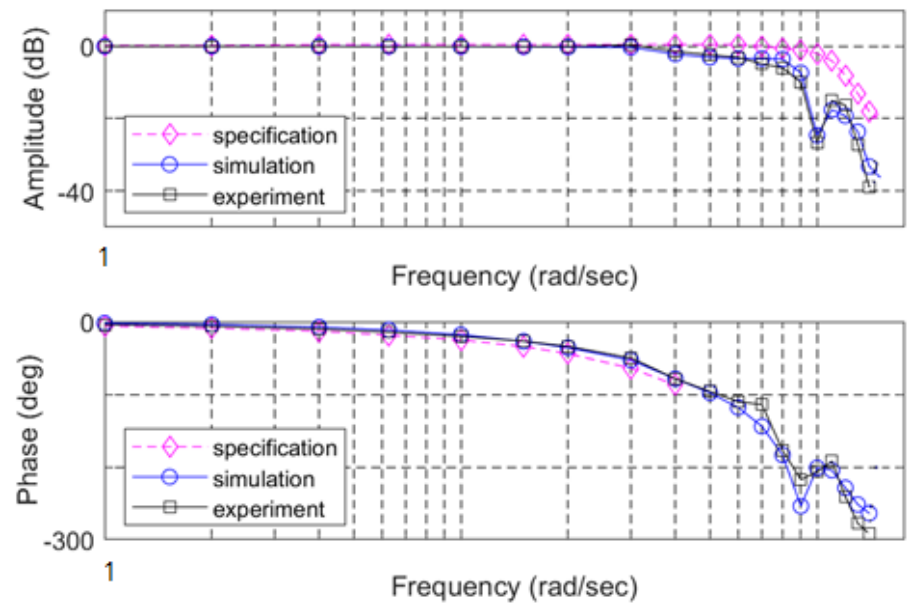

Figure 10. The final output dynamics of the compensated electro-hydraulic actuation system with a liquid-hydrogen-liquid-oxygen engine as load

With the combined control algorithm, the final compensated load frequency response $X_{L}$ is show $n$ in Fig. 10, where the open loop gain was increased to $25 \mathrm{rad} / \mathrm{s}$. The parameters in the PID and feedforward compensation were optimized around the nominal point. The response data at the marked frequencies in Fig.10 are listed in Tab.2.

TABLE 2. The compensated load dyna mic response at marked frequencies

\begin{tabular}{ccc}
\hline Frequency(rad/s) & Amplitude(dB) & Phase(degree) \\
\hline 1 & 0.00 & -4.03 \\
2 & 0.00 & -6.07 \\
4 & -0.02 & -10.02 \\
6.28 & -0.10 & -14.28 \\
10 & -0.05 & -20.02 \\
15 & -0.06 & -26.72 \\
20 & -0.08 & -34.02 \\
\hline
\end{tabular}




\begin{tabular}{ccc}
\hline Frequency $(\mathrm{rad} / \mathrm{s})$ & Amplitude(dB) & Phase(degree) \\
\hline 30 & 0.40 & -49.86 \\
40 & -1.36 & -78.36 \\
50 & -2.36 & -96.05 \\
60 & -3.07 & -109.89 \\
70 & -4.85 & -114.13 \\
80 & -5.93 & -177.13 \\
90 & -9.97 & -217.44 \\
100 & -26.61 & -206.48 \\
110 & -15.03 & -192.02 \\
120 & -16.27 & -240.83 \\
130 & -27.10 & -277.58 \\
140 & -38.99 & -291.92 \\
\hline
\end{tabular}

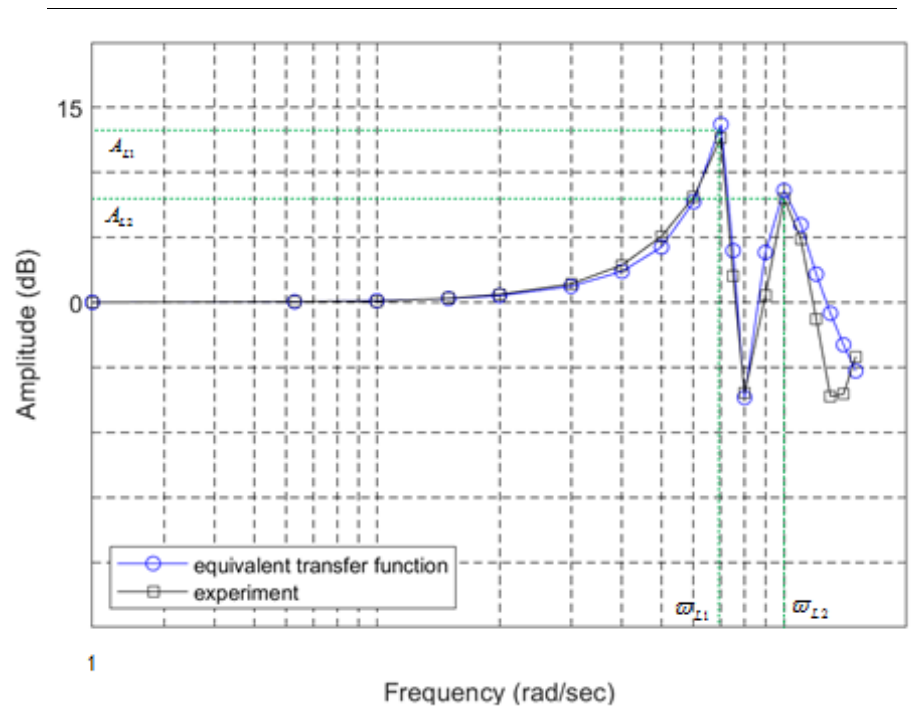

Figure 11. The structural dyna mics of a liquid-oxygen-ke rosene launcher engine

\section{A Modified Model and its Control Strategy for A More Complex System}

While a one-mass-one-spring model is sufficient for most aerospace loads, there has to be some modifications for a high thrust liquid-oxygen-kerosene engine since it has a more complicated mass and stiffness distribution [11]. It has two apparent structural natural frequencies so that a two-masstwo-spring model has to be used. The tested structuraldynamics $w$ as plotted and compared with the simulation in Fig. 11. The two-mass-two-spring physical model is depicted in Fig.12.

The corresponding normalized system block diagram was derived as Fig.13, where there are similar representations as in Fig.5 but with an additional structural resonance and an additional corresponding composite hydro-mechanical resonance. Since the two structural resonance frequencies are near each other, neither can be ignored and both have to be treated with carefully.

As in the control algorithm as in Fig.6, tw o rather than one notch filters were used as Eq. (19).

Again with the combined control algorithm, the satisfactory dynamics was obtained as in Fig. 14.

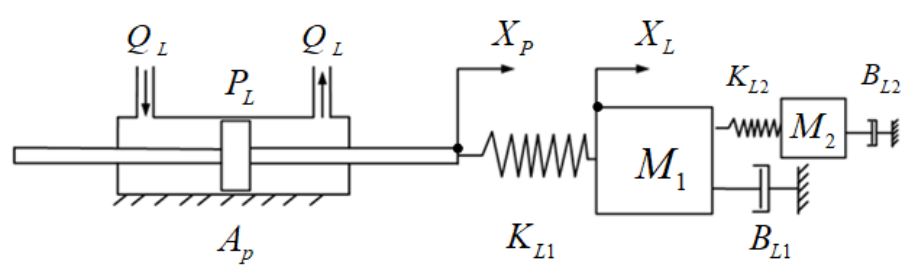

Figure 12. The physical model of a hydraulic actuator to drive a liquid-oxygen-kerosene engine 


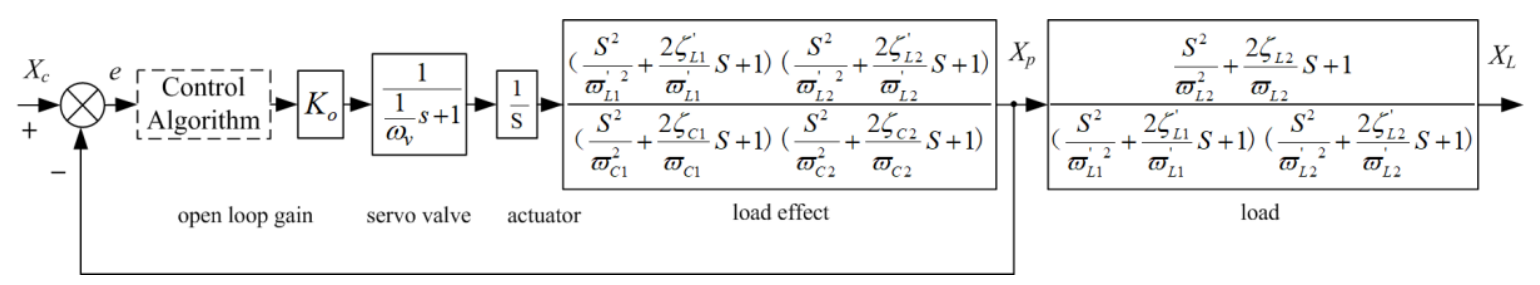

Figure 13. The normalized system block diagram to drive a two-mass-two-spring load

$$
G_{n}=\frac{\frac{1}{\omega_{n 1}^{2}} S^{2}+\frac{2 \xi_{n 1}}{\omega_{n 1}} S+1}{\frac{1}{\omega_{n 2}^{2}} S^{2}+\frac{2 \xi_{n 2}}{\omega_{n 2}} S+1} \cdot \frac{\frac{1}{\omega_{n 3}^{2}} S^{2}+\frac{2 \xi_{n 3}}{\omega_{n 3}} S+1}{\frac{1}{\omega_{n 4}^{2}} S^{2}+\frac{2 \xi_{n 4}}{\omega_{n 4}} S+1}
$$
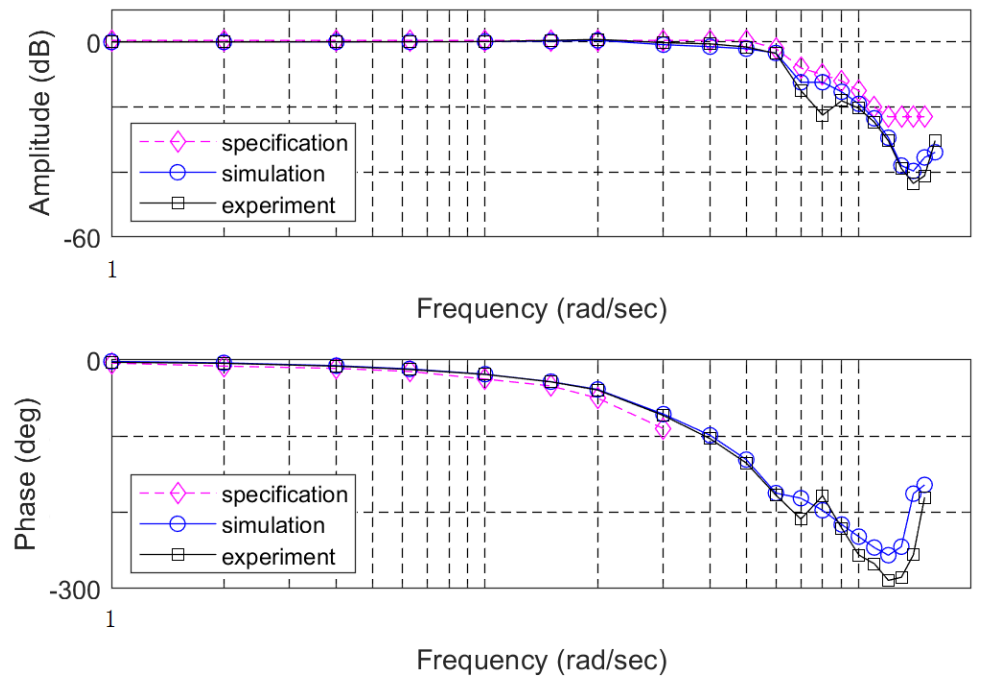

Figure 14. The final output dynamics of the compensated electro-hydraulic actuation system with a liquid-oxygen-ke rosene engine as load

\section{Discussions}

As shown above, different from some applications where only the hydraulic resonance is cared, the load structural resonance cannot be ignored in an aerospace electro-hydraulic actuation system demanding as fast as possible. There is no reason that, in a highly dynamic actuator, the hydraulic natural frequency given by the design should be lower than that of the controlled target. Therefore, the load structural natural frequency becomes the low est in the system and is the dominating design constraint.

One may argue that, there should be no the structural natural frequency $\omega_{L}$ part if the feedback is taken at the engine angular output point, as can be easily seen in Fig.4 and Fig.5, in that it would be cancelled out. Nonetheless, there is the composite hydro-mechanical part left with the natural frequency $\omega_{c}$. Because $\omega_{c}$ is derived from $\omega_{L}$ and $\omega_{h}$ as in Eq. (10), it is clear that the structural natural frequency $\omega_{L}$ is still the lower and the ceiling to limit dynamics.

Since the demanded system bandwidth is approaching the structural natural frequency, a combined control algorithm has to be carefully designed, here comprising a notch filter to suppress the resonance in the high frequency region, a feedforward compensation to improve the phase margin in the intermediate frequency region, and a nonlinear PID to upgrade the performance in the low frequency region, respectively. Fortunately, a digital control approach was applied and the seemingly complicated algorithm can be easily implemented in softw are. Even, in some applications, more complicated PID algorithms were used, e.g., an exponential proportional gain in the null region.

With the digital convenience, the parameters in the control algorithm can be defined to be changed easily as different actuators or engines used. With a same software package, a couple of 
actuation systems, e.g., that to drive liquid-oxygen-kerosene engines and liquid-hydrogen-liquidoxygen engines, have completed a couple of flight missions.

Moreover, compared to the traditional DPF method in which electronic or hydro-mechanical devices to sense the differential pressures are needed, the digital approach is more effective, economical, flexible and reliable, due to the critical hardw are reduced.

Lastly, though in the presented systems there are servo-valves used, the approach applies to pump controlled systems in fast moving applications, i.e., electro-hydrostatic actuators (EHA), in which the hydraulic cylinders are used as well.

\section{Conclusions}

The load structural resonance w as measured and represented clearly in a Bode plot. The concise second-order and fourth transfer function models were constructed, representing a one-mass-one spring model and a two-mass-two-spring model, respectively. A normalized system model was developed, comprising a closed loop for the piston displacement plus a load structural resonance outside. Inside the loop, the composite hydro-mechanical resonance w as modelled and identified by the experiment. A combined control strategy was used to obtain satisfactory dynamic performances in the whole frequency range from low to high. The experiment data matched well with the simulations and satisfactory performance were obtained. It is demonstrated that, in a highly dynamic aerospace electro-hydraulic actuation system, the load structural resonance, rather than the hydraulic resonance as often stated in other applications, is the dominating constraint and should be treated in the foremost place in the design.

Acknowledgments: The research has been funded by the Chinese non-toxic-non-pollution launch vehicle programs. This pa per publication is supported in part by the specific program “Manufacturing Basic Technology and Critical Components" of National Key Research and Development Program of China (No. 2019YFB2005101).

Author Contributions: Mr. Zhao Shoujun put forward the methods to measure the load structural re sonance and constructed the fra mework of the normalized model and the control algorithm. Mr. Chen Keqin conducted the simulation and experiments and decided the specifics of a feedforward compensation. Ms. Zhang Xiaosha built the original mathematical models and guided the simulation, experiments and substantiation of detailed control algorithms for a variety of actua tion systems. Dr. Zhao Yingxin optimized the model parameters of the structural dynamics and implemented the control algorithms for actuators to gimbal liquid-oxygen-ke rosene engines. Mr. Jing Guanghui fina lized the PID parameters and implemented the control a lgorithms for actuators to gimbal liquid-hydrogen-liquid-oxygen engines. Mr. Yin Chuanwei developed the all-factor optimization computer program for the control parameters and constructed the algorithm framework for digital signal processor based controllers. Ms. Xia oxue contributed to the data analysis and experiments.

Conflicts of Interest: The authors decla re no conflict of interest.

\section{Abbreviations}

The following abbreviations are used in this manuscript:

DPF: Dynamic Pressure Feedback

PID: Proportional, Integral and Differential

EHA: Electro-hydrostatic Actuator

\section{Appendix A}

The following mathematical symbols are used in this manuscript:

$S$ : Laplace operator;

$Q_{L}$ : the loaded flowrate to the actuator;

$A_{p}$ : the acting piston area;

$X_{c}$ : the normalized command signal; 
$X_{p}$ : the normalized piston position;

$X_{L}$ : the normalized load position output of the engine in the e quivalent linear form;

$V_{t}$ : the total controlvolume of the two actua tor chambers;

$\beta$ : the equivalent bulk modulus of the contained oil;

$P_{L}:$ the differential pressure across the piston;

$K_{c}$ : the lumped leakage coefficient across the piston, including that of the servovalve;

$K_{L}$ : the equivalent line arload stiffness;

$M$ : the equivalent moving load mass;

$B_{L}$ : the linear damping coefficient of the gimbaling engine;

$J$ : the rotationalload inertia;

$R$ : the rotation radius of the load;

$\omega_{L}, \xi_{L, \text {, }}$ the structural natural fre quency and corresponding damping ratio;

e: the normalize position error between $X_{c}$ and $X_{p}$;

$\omega_{h}, \xi_{h,}$ : the hydraulic natural fre quency and corresponding damping ratio;

$\omega_{c}, \xi_{c,}$ : the composite hydro-mechanical natural fre quency and corresponding damping ratio;

$\omega_{L 1}, \xi_{L 1}, \omega_{L L 2}, \xi_{L 2}$ : the first and second structural natural frequencies and corresponding damping ratios for a complex liquid-oxygen-ke rosene engine;

$\omega_{L 1}^{\prime}, \quad \xi_{L 1}^{\prime}, \omega_{L 2}^{\prime}, \quad \xi_{L 2}^{\prime}$ : the first and second a ppa rent structural natural frequencies and corresponding damping ratios for a complex liquid-oxygen-ke rosene engine, de rived from $\omega_{L 1}, \xi_{L 1}, \omega_{L 2}, \xi_{L 2}$;

$\omega_{c 1}, \xi_{c 1}, \omega_{c 2}, \xi_{c 2}$ : the two composite hydro-mechanical natural frequency and corresponding damping ratio for an electro-hydraulic actuation system to drive a complex liquid-oxygen-kerosene engine;

$\omega_{v}$ : the first order servovalve fre quency bandwidth;

$K_{o}$ : the nominalopen loop gain of the piston position loop;

$K v i$ : the volt-to-current conversion coefficient of the servovalve coil driver;

$K_{q i}$ : the nominal se rvovalve flowgain;

$K_{p d}$ : the conversion coefficient of the piston displacement sensor;

$K_{p}$ : the nominal proportional gain;

$K_{i}$ : the integralgain;

$K_{d}$ : the differentialgain;

$f_{k}$ : the bigger proportional gain of the piece wise proportional gain;

$K_{f}$. the feedforward compensation gain;

$A$ : amplitude in the bode plot

$\theta$ : phase lag in the bode plot

$C_{p}$ : the closed-loop frequency response of the piston position represented in the complex form

$C_{P o}$ : the open loop frequency response of the piston position represented in the complex form

$\omega_{n 1}, \xi_{n 1}, \omega_{n 2}, \xi_{n 2}$ : the compensation notch filter and its numerator and denominator frequencies and damping ratios, respectively;

$\omega_{n 1}, \xi_{n 1}, \omega_{n 2}, \xi_{n 2}$ : the 2 nd compensation notch filter and its numerator and denominator frequencies and da mping ratios, respectively;

\section{References}

1. Herbert E. Merritt. Chapter V, Hydraulic Power Element. In Hydraulic Control Systems. Copyright 1967 by John Wiley@Sons, Inc. USA. 1967.

2. John W. Edwards. Analys is of an ele ctro-hydraulic aircraft control surfa ce servo and compa rison with test results. NASA Technical Note, NASA TN D-6928. Washington D.C. August 1972.

3. Karl-Erik Rydberg. Hydraulic servo systems. IEI / Fluid and Mechanical Engineering Systems. Linköpings Universitet. 2008-10-15

4. Dragan V. Lazic'. Milan R. Ristanovic'. Electrohydraulic thrust vector control of twin rocket engines with position feedback via angular transducers. Control Engineering Practice 15 (2007) 583-594.

5. Mohit Law. Design Guidelines for an Electro-Hydraulic Actuator to Isolate Machines from Vibrations. $6^{\text {th }}$ International \& 27 th All India Manufacturing Technology, Design and Research Confe rence (AIMTDR2016), December 16-18, 2016, at College of Engineering, Pune, Maharashtra, INDIA 
6. Yang Li. Zongxia Jiao. Zimeng Wang. Design, Analysis, and Verification of an Electro-Hydrostatic Actuator for Distributed Actua tion System. Sensors 2020, 20, 634; doi:10.3390/s20030634

7. Zhihui LI. Yaoxing SHANG *. Zongxia JIAO. Yan LIN. Shuai WU. Xiaobin LI. Analysis of the dynamic performance of an electro-hydrostatic actua tor and improvement methods. Chine se Journal of Ae ronautics, (2018), 31(12): 2312-2320. https://doi.org/10.1016/j.cja.2018.03.014.

8. Zeng Guangshang. Zhang Xiaosha. A TVC system for liquid oxygen-kerosene launch vehicle. 57th International Astronautical Congress, IAC-06-C4.1.06, Hyderabad, India. 24-28 Se pt. 2006

9. Zhao Shoujun. Zhao Yingxin. Zhang Xia osha. The TVC Systems for a Chinese Liquid Oxygen and Ke rosene Launch Vehicle, IAC-13-C4,P,27.p1, 64th Inte mational Astronautical Congress (IAC), Beijing, China, 25-29 Oct. 2013

10. Guanghui Jing. Xiaosha Zhang. Guangshang Zeng. Shoujun Zhao. Chuanwei Yin. A HydrogenTurbopump-Powered Thrust Vector ControlServo System for High Thrust LH2/LOX Rocket Engines, IAC17-D2.5.10, 68th International Astronautical Congress (IAC), Adelaide, Australia, 25-29 Se pt. 2017

11. Yin Chuanwei. Zha o Shoujun. Chen Keqin. "The Algorithm Optimiza tion Study on the Thrust Vector Servo Control for a Rocket Engine with Two Resonant Frequencies (in Chinese)", Rocket and Launch Vehicle Technology, 1(324):21-26, 2013

(C) 2020 by the authors; licensee MDPI, Basel, Switzerland. This article is an open access article distributed under the terms and conditions of the Creative Commons by Attribution (CC-BY) license (http://creativecommons.org/licenses/by/4.0/). 